\title{
Replication of Uniformly Accessed Shared Data for Large-Scale Data-Parallel Algorithms*
}

\author{
Chung-Ming Chen \\ Center for Biomedical Engineering \\ National Taiwan University \\ Taipei, Taiwan, R.O.C.
}

\author{
Soo-Young Lee \\ Department of Electrical Engineering \\ Auburn University \\ Auburn, AL 36849
}

\begin{abstract}
In this paper, we show how to minimize data sharing overhead required in most parallel algorithms, especially in Large-Scale Data-Parallel (LSDP) algorithms on a $2 D$ mesh. Two specific issues are addressed in this study. One is what the optimal group size is, i.e., how many PEs should share a copy of shared data. The other is where the replicated data should be allocated.
\end{abstract}

\section{Introduction}

Data sharing is inevitable in most parallel algorithms. For many problems, especially, for those problems with rich data parallelism, the overhead due to data sharing, i.e., data movement or data access contention $[1]$, is one of the major factors degrading the performance of a parallel algorithm.

In this study, we investigate how to minimize the overhead caused by data sharing for a class of problems modeled as the Large-Scale Data-Parallel (LSDP) algorithms on a wrapped-around and a regular 2D meshes. An LSDP algorithm has the following features: (i) it has rich data-parallelism but without exclusive task and data partitioning, which means that some data need to be shared by multiple PEs, (ii) a great amount of shared data is involved (therefore, communication overhead is high), (iii) a synchronization point is required before any use of the shared data. This computation model may be found as the only or one of the major algorithmic structures in many applications, e.g., in an EM reconstruction algorithm for 3D Positron Emission Tomography [2].

Minimization of data sharing overhead for an LSDP algorithm is attempted by optimizing data access patterns in this study. A data access pattern specifies when and where to access shared data for each PE. Once all tasks have been assigned to PEs, the data sharing overhead is mainly determined by the data arcess pattern. In this paper, a uniform data access pattern is assumed, i.e., every PE has the same number of accesses to each of all other PEs. Our approach is to optimally replicate the shared data and allocate the replicated data. We also develop scheduling algorithms specifying shared data access sequences to

*This work was supported in part by grant number R01 CA51324 from the National Cancer Institute, NIH.

The authors were with School of Electrical Engineering, Comell University achieve the minimal (optimal) communication overhead.

Data replication is a widely used technique to enhance data locality at the expense of integrating and broadcasting replicated data. However, optimizing data replication has not been attempted in most previous works.

\section{Models}

\subsection{System Model}

The system topology to be considered in this paper is a wrapped-around and a regular (non-wrappedaround) 2D meshes with $N \times N$ PEs. Refer to [4] for the results on a hypercube.

A system is bi-directional if its links (i.e., the connections between adjacent PEs) are capable of realizing communication in both directions simultaneously. If each link of a system can perform communication in both directions but only one direction at a time, this system is considered as a uni-directional system. We assume that all links can perform communication independently.

In this study, analysis on a 2D mesh is based on the analysis on a linear array or a ring. For convenience, we use $W S_{i}, i=1,2$, to denote uni- and bi-directional wrapped-around $2 \mathrm{D}$ meshes, where the value of $i$ indicates the number of directions realizable at a time by a link. Similarly, we use $R S_{i}, i=1,2$, to denote uniand $b i$-directional regular $2 \mathrm{D}$ meshes, respectively.

Throughout this paper, we use the following convention to label PEs and links. For a ring or a linear array with $m$ PEs, $p_{i}$ denotes the $i$ th $\mathrm{PE}$, where $0 \leq i \leq(m-1)$ and $l_{i}$ denotes the left link of $p_{i}$, where $0<i<(m-1)$. For a wrapped-around or a regular $2 \mathrm{D}$ mesh with $m$ rows and $n$ columns of PEs, $p_{i j}$ denotes the PE at the $i$ th row and the $j$ th column, $h l_{i j}$ denotes the left (horizontal) link of $p_{i j}$, and $v l_{i j}$ denotes the top (vertical) link of $p_{i j}$, where $0 \leq i \leq(m-1), 0 \leq j \leq(n-1)$. As an example, link labeling of a $4 \times 3(m=4$ and $n=3)$ wrapped-around 2D mesh is illustrated in Fig. 1, where the circle with a number $i j$ inside represents $p_{i j}$.

\subsection{Computation Model}

Let $M$ denote the total number of shared data, $N_{a}$ the average number of accesses to each shared datum 
for each PE and $S_{M}$ the total number of shared data accesses by each PE which is equal to $N_{\mathrm{a}} \times M$.

The computation model employed in this study, the Large-Scale Data-Parallel (LSDP) algorithm, consists of a large number of data-parallel computations. With multiple $\mathrm{PEs}$, it is assumed that each $\mathrm{PE}$ performs a set of data-parallel computations beginning and ending with a synchronization point, respectively. Each data-parallel computation produces a partial result for a shared datum.

All PEs are divided into groups and all groups have the same number of PEs. Let $n_{g}$ denote the group size, i.e., the number of PEs in a group. The shared data are replicated such that a copy of replicated data shared (which may be a subset of the entire shared data) by a group of PEs is evenly distributed among these PEs. All computations and associated shared data accesses are distributed over $N_{b}$ pairs of computation and communication bands. Within each computation band, all PEs perform the same number of data-parallel computations.

To make the cost for each computation band independent of data replication for ease of analysis, all partial results computed by a $\mathrm{PE}$ are temporarily stored in the local memory of the PE. In the following communication band, each PE sends those partial results of which associated shared data are not in its local memory to the PEs which hold the associated shared data. Then, each PE modifies the shared data in its local memory using the partial results at the end of each communication band.

The overall time required for each communication band, defined as updating time and denoted by $T_{u}$, may be decomposed into two components. One component, denoted by $T_{u m}$, is the time for all PEs to perform modifications using the partial results. The other component, denoted by $T_{u x}$, is the time required for all PEs to send the partial results to all other PEs in the same group.

If the shared data are replicated, before the synchronization point at the end, the (modified) replicated data in all PEs are integrated to ensure data coherence. Also, the integrated shared data are broadcast to all PEs. Note that the size of shared data involved in a broadcasting is $n_{g}$ times larger than that in an integration.

The overall time required for integrating and broadcasting shared data is defined as integration and broadcasting time. Like the updating time, the integration and broadcasting time, denoted as $T_{I}$ is composed of two parts. One part, denoted as $T_{I x}$, is the time for transmitting data and the other part, denoted as $T_{I_{m}}$, is the time for performing the modifications on the replicated data. We use the integration and broadcasting algorithms proposed in [2]. The time required by the integration and broadcasting with $N_{I}$ PEs is $\mathcal{O}\left(\left(1-1 / N_{I}\right) M\right)$ using the integration and broadcasting algorithms [2].

The goal of this study is to minimize the data sharing overhead, including updating time as well as integration and broadcasting time. It needs to be noted that all time measurements used in the following discussions are normalized by the time to transfer one datum between two adjacent PEs during integration and broadcasting. Let $T_{m}$ denote the time required for one modification and $T_{x}$ the time required for transferring one datum between two adjacent PEs in a communication band.

\section{Data Replication}

In the following, we first discuss how the replicated data should be allocated. Then, the optimal group size is derived for the best type of allocation.

Due to the limited space, most of the propositions will be given without proofs. Refer to [4] for the proofs.

\subsection{Two Types of Data Allocation}

Two types of allocations may be considered for replicated data, namely, aggregate and scatter, as illustrated in Fig. 2 for a $2 \mathrm{D}$ mesh. If all PEs in each group, represented in the same pattern in Fig. 2 form a continuous region, it is called the aggregate type allocation. If each group is further divided into subgroups of the same size and the corresponding subgroups of all groups form a cluster as shown in Fig. 2, it is called the scatter type allocation.

For a $2 D$ mesh, let the group size be $x \times y$ where $y>x$ and $N$ is divisible by $x$ and $y$. In this example (Fig. 2), PEs are divided into 9 groups indicated by 9 different patterns, each group with 4 PEs.

With a uniform data access pattern, the size of each packet of data, denoted as $s$, is equal to $N_{a} M /\left(N_{b} x y\right)$ on a $2 \mathrm{D}$ mesh.

We have performed a detailed formal comparison between the two allocations [4], which cannot be presented here due to the limited space. It has been shown under our system and computation models that the aggregate type allocation is not worse than the scatter type allocation.

\section{2 $T_{u x}$ and $T_{I x}$ with The Aggregate Type Allocation on A Mesh}

To derive the optimal $T_{u x}$ for $R S_{1}, R S_{2}, W S_{1}$ and $W S_{2}$ types of systems for the aggregate type allocation, we first compute the optimal $T_{u x}$ for the 1D case and then extend the results to the $2 \mathrm{D}$ case. The general procedure to prove the optimality of $T_{u x}$ and $T_{I x}$ for each system is to first find out the lower bound of the optimal $T_{u x}$ and $T_{Y x}$, respectively, and then to show that these lower bounds are achievable by designing a scheduling algorithm.

\subsubsection{Optimal $T_{u x}$ for a linear array}

Suppose that there are $x$ PEs in a linear array. The optimal $T_{u x}$ is derived in Proposition 3.1.

Proposition 3.1 The optimal $T_{u x}$ on a linear array is $\beta\left[\left(x^{2}-1\right) / 4\right\rceil s T_{x}$, where $\beta$ is 1 and 2 for $a$ bi-directional and a uni-directional linear arrays, respectively.

$1 D$ Updating Algorithm for a uniform DAP on a linear array

1. At step $0, p_{0}$ sends different packets of data to all other PEs in the sequence of $p_{x-1}, p_{x-2}, \ldots$, $p_{1}$. The size of each packet is $s$. While the data 
from $p_{0}$ to $p_{x-1}$ are being sent, all other PEs except $p_{x-1}$ also send a packet of data to $p_{x-1}$ in parallel.

2. At step $i$, where $i<\lceil x / 2\rceil, p_{i}$ sends a different packet of data to each remaining $P E$ in the sequence of $p_{x-1-i}, p_{x-2-i}, \ldots, p_{i+1}$. While the data from $p_{i}$ to $p_{x-1-i}$ are being sent, all $p_{k}, i<k<(x-1-i)$, also send a packet of data to $p_{x-1-i}$ in parallel.

This algorithm only describes the scheduling from left to right. Data accesses from right to left may be performed by using this algorithm but in a reversed direction.

\subsubsection{Optimal $T_{u x}$ for $R S_{1}$ and $R S_{2}$}

On a regular 2D mesh with $x$ rows and $y$ columns of PEs and $y \geq x$, each datum is sent either horizontally followed by vertically or vertically followed by horizontally. Therefore, transferring data for updating on a regular $2 \mathrm{D}$ mesh is basically composed of two data transfers, each on linear arrays.

Proposition 3.2 The optimal $T_{u x}$ on a regular mesh with $x \times y$ PEs, is $\beta x\left\lceil\left(y^{2}-1\right) / 4\right\rceil s T_{x}$, where $y \geq x$ and $\beta$ is 1 and 2 for an $R S_{2}$ and an $R S_{1}$ systems, respectively.

$2 D$ Updating Algorithm for a uniform DAP on a regular $2 D$ mesh

1. Divide each packet of data into two subpackets, namely, the first-half and the second-half subpackets, each with $s / 2$ data.

2. Each row and column of PEs perform $1 D U p$ dating Algorithm for a uniform $D A P$ on a linear array twice for the corresponding linear arrays.

- In the first phase, for all $i$ and $j, p_{i j}$ combines all first-half subpackets for all $p_{a k}, a=$ $0, \ldots, x-1$ and $k \neq j$, into a superpacket and sends it to $p_{i k}$ along the $i$ th row. Also, $p_{i j}$ combines all second-half subpackets for all $p_{k b}, b=0, \ldots, y-1$ and $k \neq i$, into a superpacket and sends it to $p_{k j}$ along the $j$ th column.

- In the second phase, for all $i$ and $j, p_{i j}$ takes out the $k$ th first-half subpackets of all superpackets received from the $i$ th row, puts them in a new superpacket, and sends it to $p_{k j}$ along the $j$ th column. Also, $p_{i j}$ takes out the $k$ th second-half subpackets of all superpackets received from the $j$ th column, puts them in a new superpacket, and sends it to $p_{i k}$ along the $i$ th row. The time required for shuffling subpackets is assumed to be negligible.

\subsubsection{Optimal $T_{u x}$ for a ring}

As for a regular mesh, to derive the optimal $T_{u x}$ for a wrapped-around mesh, we first derive that for a ring. Consider a ring with $x \mathrm{PEs}$. For sending data from $p_{i}$ to $p_{j}$, define left-distance as the number of links in the left-path, $\left\{p_{i}, p_{(i-1+x) \bmod x}, \ldots, p_{j}\right\}$, and define rightdistance as that in the right-path, $\left\{p_{i}, p_{(i+1) \bmod x}, \ldots\right.$, $\left.p_{j}\right\}$. To fully utilize the wrapped-around feature, each packet is sent through the path of $\min \{$ left-distance, right-distance $\}$. If the left-distance is equal to the right-distance for a packet, this packet is split into two subpackets and each of the left-and right-paths carries one subpacket. Therefore, the maximal distance between every send-receive pair of PEs is $[x / 2]$.

Proposition 3.3 The optimal $T_{u x}$ on a ring is $\beta\left[\left(x^{2}-1\right) / 4\right] s T_{x} / 2$, where $\beta$ is 1 and 2 for $a$ bidirectional and a uni-directional rings, respectively.

\subsubsection{Optimal $T_{u x}$ for $W S_{1}$ and $W S_{2}$}

Like for a regular mesh, for a rectangular wrappedaround mesh with $x$ rows and $y$ columns of PEs and $y \geq x$, transferring data is also composed of two data transfers, each on rings.

Proposition 3.4 The optimal $T_{u x}$ on a wrappedaround mesh with $x \times y P E s$, is $\beta x\left\lceil\left(y^{2}-1\right) / 4\right\rceil s T_{x} / 2$, where $y \geq x$ and $\beta$ is 1 and 2 for an $W S_{2}$ and an $W S_{1}$ systems, respectively.

For comparison, in [3], the optimal $T_{u x}$ has been shown to be $\Theta\left(p^{2 / 3}\right)$ for a square wrapped-around mesh with $p \times p$ PEs.

Although a group (a submesh) in a wrappedaround 2D mesh is a regular mesh, the updating algorithm for a wrapped-around 2D mesh may be used by each group due to the wrapped-around feature of the whole mesh. Each submesh can actually be considered as a wrapped-around submesh by incorporating the rule: each PE in a group, without loss of generality, communicates with $[y / 2\rfloor$ PEs to its right and $y-\lfloor y / 2\rfloor-1$ PEs to its left on its row as well as $\mid x / 2\rfloor$ PEs above it and $y-\lfloor x / 2\rfloor-1$ PEs below it on its column. As a result, each submesh has the same optimal $T_{u x}$ as if it were a wrapped-around submesh.

\subsubsection{Optimal $T_{I x}$ for $R S_{1}$ and $R S_{2}$}

For a regular $2 \mathrm{D}$ mesh, integration and broadcasting are done through a ring communication pattern. It is assumed that all corresponding PEs among groups on a regular $2 \mathrm{D}$ mesh can form a ring.

As an example, Fig. 3 illustrates one of the rings for integration. There are $N^{2} /(x y)$ PEs involved in the integration in each ring. It is easy to see that some links are shared by $y$ rings, e.g., the central link on the first row in Fig. 3. It means that the data transferred in these $y$ rings will pass through this central link during integration. The effective size of data passing through this central link in each ring is $\left(1-x y / N^{2}\right) s$ [2], where $s$ is the packet size which is $M /(2 x y)$ for an $R S_{2}$ system and $M /(x y)$ for an $R S_{1}$ system. Therefore, the time required by the integration is $y\left(1-x y / N^{2}\right) s T_{x}$. For broadcasting, all PEs are involved in the same broadcasting and the packet size is $M / 2 N^{2}$ and $M / N^{2}$ for an $R S_{2}$ and an $R S_{1}$ system, respectively. As a consequence, by using the Linear 
integration and broadcasting algorithm, the optimal $T_{I x}$ normalized by $M$ for a regular mesh, denoted as $T_{I x_{r}}$, is

$$
T_{I_{x_{r}}}=\frac{\beta}{2}\left(\frac{1}{x}\left(1-\frac{x y}{N^{2}}\right)\right)+\frac{\beta}{2}\left(1-\frac{1}{N^{2}}\right)
$$

where $\beta=1$ for an $R S_{2}$ system, $\beta=2$ for an $R S_{1}$ system.

\subsubsection{Optimal $T_{I x}$ for $W S_{1}$ and $W S_{2}$}

For a wrapped-around 2D mesh, a ring pattern may be used for integration and broadcasting in each row and column. To fully utilize the wrapped-around characteristic of $W S_{2}$ and $W S_{1}$ systems, during integration, the data to be integrated are divided into two parts with sizes of $s_{1} s$ and $s_{2} s$ satisfying $s_{1}+s_{2}=1$ where $s=M /(x y)$. While one part is integrated vertically followed by horizontally, the other part may be integrated horizontally followed by vertically. Similarly, during broadcasting, each packet is divided into two parts of the same size, i.e., $M / 2 N^{2}$. For a $W S_{2}$ system, each part may be further divided into two subparts of the same size, which are sent in two opposite directions of a ring. Then, normalized by $M$,

$$
\begin{aligned}
T_{I x} & =\max \left\{\frac{\beta s_{1}}{2 y}\left(1-\frac{x}{N}\right), \frac{\beta s_{2} y}{2 x}\left(1-\frac{y}{N}\right)\right\} \\
& +\max \left\{\frac{\beta s_{1}}{2 N}\left(1-\frac{y}{N}\right), \frac{\beta s_{2}}{2 N}\left(1-\frac{x}{N}\right)\right\} \\
& +\frac{\beta}{4}\left(1-\frac{1}{N^{2}}\right) .
\end{aligned}
$$

Proposition $3.5 T_{I x}$ for a wrapped-around $2 D$ mesh is minimized when $\left(s_{1}, s_{2}\right)=\left(s_{11}, s_{21}\right)$, where

$$
\begin{aligned}
& s_{11}=\frac{y(N-y)}{y(N-y)+x(N-x)} \\
& s_{21}=\frac{x(N-x)}{y(N-y)+x(N-x)}
\end{aligned}
$$

Therefore, the optimal $T_{I x}$ normalized by $M$ for a wrapped-around mesh with a group size of $x \times y$, denoted as $T_{I x_{w}}$, is

$$
\begin{array}{ll}
\frac{\beta s_{1}}{2}\left(\frac{1}{y}\left(1-\frac{x}{N}\right)+\frac{1}{N}\left(1-\frac{y}{N}\right)\right)+\frac{\beta}{4}\left(1-\frac{1}{N^{2}}\right) & \text { if } S_{1} \geq S_{2} \\
\frac{\beta s_{2}}{2}\left(\frac{1}{x}\left(1-\frac{y}{N}\right)+\frac{1}{N}\left(1-\frac{x}{N}\right)\right)+\frac{\beta}{4}\left(1-\frac{1}{N^{2}}\right) & \text { if } S_{1}<S_{2}
\end{array}
$$

where $\beta=1$ for a $W S_{2}$ system and $\beta=2$ for a $W S_{1}$ system.

\subsection{Optimal Data Replication on A Mesh}

In this section, we would like to determine the optimal group size for data replication with a uniform data access pattern, using the aggregate type allocation.

Let $T_{r}$ denote the overall data sharing time, normalized by $M$ and the time for transferring one datum between adjacent PEs, for a regular 2D mesh with a group size of $x \times y$. Recall that the overall data sharing time is $N_{b}\left(T_{u m}+T_{u x}\right)+T_{I m}+T_{I x}$, where $T_{u m}, T_{u x}, T_{I m}$ and $T_{I x}$ have been derived in the previous sections. Then,

$$
T_{r}=\beta\left\lceil\frac{y^{2}-1}{4}\right\rceil \frac{N_{a} T_{x}}{y}+T_{I x_{r}}+N_{a} T_{m}+\left(\frac{1}{x y}-\frac{1}{N^{2}}\right) T_{m}
$$

where $\beta=1$ for an $R S_{2}$ system, $\beta=2$ for an $R S_{1}$ system, and $T_{I_{x_{r}}}$ as defined in Eq. (1). Similarly, let $T_{w}$ denote the overall normalized data sharing time for a wrapped-around $2 \mathrm{D}$ mesh with a group size of $x \times y$,

$$
T_{w}=\beta\left\lceil\frac{y^{2}-1}{8}\right\rceil \frac{N_{a} T_{x}}{y}+T_{I x_{w}}+N_{a} T_{m}+\left(\frac{1}{x y}-\frac{1}{N^{2}}\right) T_{m}
$$

where $\beta=1$ for a $W S_{2}$ system, $\beta=2$ for a $W S_{1}$ system and $T_{I x_{w}}$ as defined in Eq. (2).

\subsubsection{The Optimal Group Size on A Mesh}

Proposition 3.6 The optimal group size for a $2 D$ mesh satisfies $x=y$.

proof:

It can be shown that $\partial T_{r} / \partial x$ and $\partial T_{w} / \partial x$ are all negative. Therefore, the optimal group size should satisfy $x=y$ since $x \leq y$.

Q.E.D.

When $x=y$, all of $T_{r}$ and $T_{w}$ reduce to

$$
\begin{aligned}
T_{o} & =\alpha\left(\left\lceil\frac{x^{2}-1}{4}\right\rceil \frac{N_{a} T_{x}}{x}+\frac{1}{2}\left(\left(\frac{1}{x}-\frac{x}{N^{2}}\right)+\left(1-\frac{1}{N^{2}}\right)\right)\right) \\
& +N_{a} T_{m}+\left(\frac{1}{x^{2}}-\frac{1}{N^{2}}\right) T_{m} .
\end{aligned}
$$

where $\alpha$, is $1,2,0.5$ and 1 for an $R S_{2}$, an $R S_{1}$, a $W S_{2}$ and a $W S_{1}$ systems, respectively. Note that Eq. (3) is also applicable when $x=y=N$. The optimal group size, $x_{\text {opt }}$, for $T_{o}$ may be determined by solving $\partial T_{o} / \partial x=0$. Suppose that $T_{o}$ is minimized at $x_{e}$ and $x_{o}$ when $x$ is even and odd, respectively. Without giving a lengthy derivation, we have the following proposition.

Proposition 3.7 Let $x_{e h}=\left\lceil x_{e}\right\rceil, x_{e l}=\left\lfloor x_{e}\right\rfloor, x_{o h}=$ $\left\lceil x_{o}\right\rceil$ and $x_{o l}=\left\lfloor x_{o}\right\rfloor$, where

$$
\begin{aligned}
& \Delta_{\text {even }} \geq 0: x_{e}=\left(\frac{2 N^{2}}{N^{2} N_{a} T_{x}-2}\right)^{\frac{1}{3}}\left(\left(\frac{2 T_{m}}{\alpha}+\Gamma_{\text {even }}\right)^{\frac{1}{3}}\right. \\
&\left.+\left(\frac{2 T_{m}}{\alpha}-\Gamma_{\text {even }}\right)^{\frac{1}{3}}\right) \\
& \Delta_{\text {even }}<0: x_{e}=2 \\
& \Delta_{\text {odd }} \geq 0: x_{o}=\left(\frac{2 N^{2}}{N^{2} N_{a} T_{x}-2}\right)^{\frac{1}{3}}\left(\left(\frac{2 T_{m}}{\alpha}+\Gamma_{\text {odd }}\right)^{\frac{1}{3}}\right. \\
&\left.+\left(\frac{2 T_{m}}{\alpha}-\Gamma_{\text {odd }}\right)^{\frac{1}{3}}\right) \\
& \Delta_{\text {odd }}<0 \quad: \quad x_{o}=1
\end{aligned}
$$




$$
\begin{aligned}
\text { and } & =54 N_{a} N^{2} T_{x} T_{m}^{2}-\alpha^{2} N^{2}-108 T_{m}{ }^{2} \\
\Delta_{\text {even }} & =432\left(N^{2} N_{a} T_{x}-2\right) T_{m}^{2}+\alpha^{2} N^{2}\left(N_{a} T_{x}-2\right)^{3} \\
\Delta_{\text {odd }} & = \\
\Gamma_{\text {even }} & =\sqrt{\frac{4 T_{m}^{2}}{\alpha^{2}}-\frac{2 N^{2}}{27\left(N^{2} N_{a} T_{x}-2\right)}} \\
\Gamma_{\text {odd }} & =\sqrt{\frac{4 T_{m}^{2}}{\alpha^{2}}-\frac{N^{2}\left(N_{a} T_{x}-2\right)^{3}}{108\left(N^{2} N_{a} T_{x}-2\right)}}
\end{aligned}
$$

The optimal group size, $x_{\text {opt }}$, for data replication with a uniform data access pattern would be $x_{o p t} \in$ $\left\{x_{e h}, x_{e l}, x_{o h}, x_{o l}\right\}$ for which $T_{o}$ is minimal.

From Proposition 3.7, we can see that for a smaller $\alpha$, the optimal group size tends to be larger. The reason is that as $\alpha$ decreases, $T_{I m}$ becomes more influential in determining the optimal group size. Since $T_{I m}$ decreases as the group size increases, a larger group size would be preferred for a smaller $\alpha$.

\section{Conclusions}

In this paper, we have presented how to optimally allocate the replicated data and what the best group sizes are for the Large-Scale Data-Parallel algorithms on a mesh with a uniform data access pattern. Also, scheduling algorithms which specify data access sequences of $\mathrm{PEs}$ for the minimal data sharing time have been developed.

The significance of these results is that given an LSDP algorithm, one can easily determine the optimal data replication and allocation, and how to control the access sequence, so that the data sharing overhead is minimized when the data access pattern is uniform. Moreover, it may serve as a guideline for parallel compilers to determine the best data distribution, for example, in parallelizing a sparse matrix computation.

\section{References}

[1] M. Gupta and P. Banerjee, "Demonstration of automatic data partitioning techniques for parallelizing compiliers on multicomputers," IEEE Trans. Parallel Distributed Syst., vol. 3, pp. 179193, Mar. 1992.

[2] C. M. Chen and S.-Y. Lee, "Parallelization of the EM algorithm for 3D PET image reconstruction: Performance estimation and analysis," in Proc. 1991 Int. Conf. Parallel Processing, vol. III, pp. 175-182, Aug. 1991.

[3] D. P. Bertsekas and J. N. Tsitsiklis, Parallel and Distributed Computation. Prentice-Hall, 1989.

[4] C. M. Chen, On Miniminzing Data Sharing Overhead for Large-Scale Data-Parallel Algorithms: Replication and Allocation of Shared Data. PhD thesis, Cornell University, Ithaca, New York, 1993.

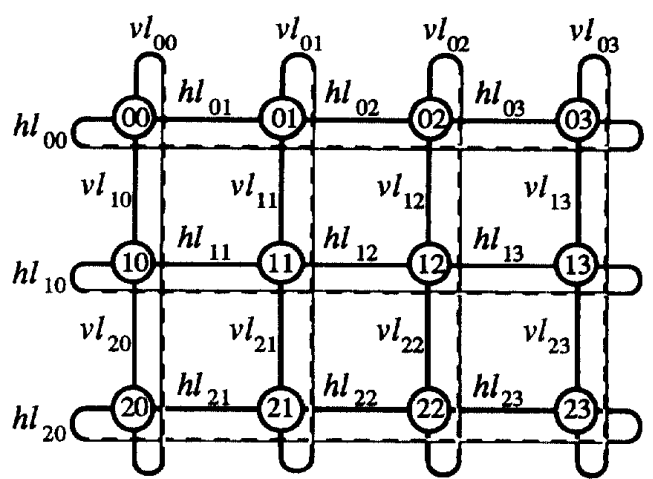

Figure 1: An illustration of the System Model

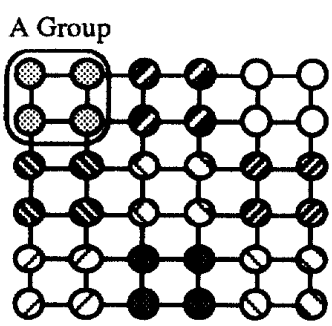

Aggregate Type

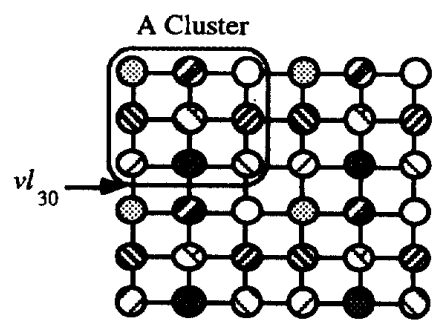

ScatterType
Figure 2: Illustrations of the aggregate and the scatter type allocations on a mesh

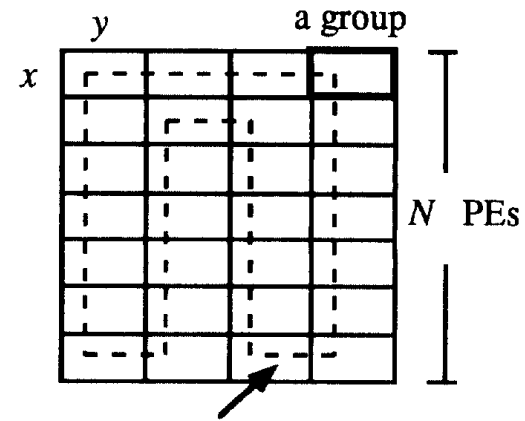

a ring for integration

Figure 3: A ring for integration and broadcasting when $N / y$ is even 